\title{
Diamond nanowires and the insulator-metal transition in ultrananocrystalline diamond films
}

\author{
R. Arenal, ${ }^{1}$ P. Bruno, ${ }^{1}$ D. J. Miller, ${ }^{1}$ M. Bleuel, ${ }^{2}$ J. Lal, ${ }^{2}$ and D. M. Gruen ${ }^{1}$ \\ ${ }^{1}$ Materials Science Division, Argonne National Laboratory, Argonne, Illinois 60439, USA \\ ${ }^{2}$ Intense Pulsed Neutron Source, Argonne National Laboratory, Argonne, Illinois 60439, USA \\ (Received 28 November 2006; revised manuscript received 22 January 2007; published 21 May 2007)
}

\begin{abstract}
Further progress in the development of the remarkable electrochemical, electron field emission, hightemperature diode, and optical properties of $n$-type ultrananocrystalline diamond films requires a better understanding of electron transport in this material. Of particular interest is the origin of the transition to the metallic regime observed when about $10 \%$ by volume of nitrogen has been added to the synthesis gas. Here, we present data showing that the transition to the metallic state is due to the formation of partially oriented diamond nanowires surrounded by an $s p^{2}$-bonded carbon sheath. These have been characterized by scanning electron microscopy, transmission electron microscopy techniques (high-resolution mode, selected area electron diffraction, and electron-energy-loss spectroscopy), Raman spectroscopy, and small-angle neutron scattering. The nanowires are 80-100 nm in length and consist of $\sim 5 \mathrm{~nm}$ wide and 6-10 nm long segments of diamond crystallites exhibiting atomically sharp interfaces. Each nanowire is enveloped in a sheath of $s p^{2}$-bonded carbon that provides the conductive path for electrons. Raman spectroscopy on the films coupled with a consideration of plasma chemical and physical processes reveals that the sheath is likely composed of a nanocarbon material resembling in some respects a polymer-like mixture of polyacetylene and polynitrile. The complex interactions governing the simultaneous growth of the diamond core and the $s p^{2}$ sheath responsible for electrical conductivity are discussed as are attempts at a better theoretical understanding of the transport mechanism.
\end{abstract}

DOI: 10.1103/PhysRevB.75.195431 PACS number(s): 71.30. + h, 81.05.Uw, 72.20. $-\mathrm{i}$, 81.07. - b

\section{INTRODUCTION}

Ultrananocrystalline diamond (UNCD) films are synthesized from argon microwave plasmas containing $1 \%$ carbon in the form of $\mathrm{C}_{60}$ or $\mathrm{CH}_{4} \cdot{ }^{1}$ Such films, composed of $3-5 \mathrm{~nm}$ randomly oriented crystallites surrounded by $0.2-0.3 \mathrm{~nm}$ wide grain boundaries, are highly electrically insulating. The progressive substitution of nitrogen for argon in the synthesis gas renders the films increasingly electrically conducting with conductivities reaching several hundred $\mathrm{S} / \mathrm{cm}$ for $20 \%$ by volume of $\mathrm{N}_{2}$ added to the synthesis gas., ${ }^{2,3}$ Hall-effect measurements have determined the carriers in these films to be $n$-type with concentrations of $\left(10^{19}-10^{21}\right) / \mathrm{cm}^{3}$ and mobilities of several $\mathrm{cm}^{2} \mathrm{~V}^{-1} \mathrm{~s}^{-1} .3,4$

These films are clearly of scientific interest but are also potentially useful, since they provide the only currently available $n$-type diamond material that is electrically conducting at ambient temperatures. One already demonstrated application of UNCD is as part of a highly rectifying diode which is electrically stable even after repeated cycling to $1300 \mathrm{~K} .{ }^{5}$ In order further to improve this use and others of this versatile material, it is important to gain a better understanding of the complex conductivity behavior of UNCD films and, in particular, to investigate in detail the mechanism underlying the insulator-metal transition. In contradiction to $p$-type diamond films whose conductivity is due to well understood substitutional doping by boron, substitutional nitrogen in the diamond lattice does not contribute at all or at best only minimally to conductivity because nitrogen occupies a doping level that lies $1.7 \mathrm{eV}$ below the conduction band. The source of the contribution of nitrogen to the conductivity of UNCD films, which is as large as that of $p$-type diamond films, cannot therefore be due to a conven- tional doping mechanism but occurs presumably by electron transport through the ubiquitous grain boundaries characteristic of nanocrystalline materials. ${ }^{6}$

The grain boundaries in UNCD are unique and have received considerable attention from theorists ${ }^{7-9}$ as well as from experimentalists. ${ }^{10-13}$ The uniqueness of UNCD grain boundaries lies in the fact that the $s p^{2}$ and $s p^{3}$ electronic configurations of carbon are exceedingly close in energy. This situation is perhaps best illustrated by the fact that only $0.02 \mathrm{eV}$ separates the free energies of formation of $s p^{2}$-bonded graphite from $s p^{3}$-bonded diamond. For this reason, energy minimization criteria leading to the formation of UNCD grain boundaries can occur in many ways. A wide variability of rehybridizations resulting in fascinating bonding configurations including highly conjugated polymer chains is a possibility that must receive careful consideration. ${ }^{14}$ Nitrogen has for a long time been known to play an important role in determining growth behavior and therefore strongly influencing the microstructure of chemical-vapor-deposited (CVD) diamond films. ${ }^{7,8}$ In the case of UNCD, nitrogen has also been found to affect the nature of the grain boundaries. ${ }^{9,11-13}$

The combination of high-resolution transmission electron microscopy (HRTEM), selected area electron diffraction (SAED), and electron-energy-loss spectroscopy (EELS) represents one of the more valuable tools for investigating the crystal structure and composition of materials at the nanometer scale. Electron-energy-loss near-edge structure (ELNES) provides key information on the local environment (chemical bonding and electronic structure) of selected elements. ${ }^{15-17}$ In the case of carbon, due to the high sensitivity of the $K$ edge, ELNES is the most powerful technique for $s p^{2} / s p^{3}$ bonding analysis at the nanometer scale. ${ }^{18}$ 
Small-angle neutron scattering (SANS) is a powerful nondestructive tool for the microscopic characterization of materials. ${ }^{19}$ SANS provides information on the size, shape, volume fraction, and interactions of particles in the size range $10-1000 \AA .{ }^{19}$ When one measures the SANS intensity around a given scattering vector $q$, one observes the structure at a spatial scale and resolution of order $q^{-1}$. We know that only spatial variations or fluctuations of density in the medium contribute to the scattering intensity. In many colloidal systems, porous media, ceramics, or suspensions, these variations of density are localized at interfaces, and then, in a certain sense, even when measuring bulk material by SANS, we are quite sensitive to interfacial phenomena. SANS provides complementary information to electron microscopy mentioned above. While the latter provides detailed information about single crystallites, the former provides statistical information about the crystallites in a large volume $\left(\sim 1 \mathrm{~cm}^{3}\right)$ and is particularly sensitive to density differences at interfaces in the given sample.

In this paper, we have studied the interplay between the effects of nitrogen on the growth patterns of UNCD crystallites with concomitant bonding pattern modifications of UNCD grain boundaries. We have brought to bear the techniques of HRTEM, EELS, SAED, scanning electron microscopy (SEM), and SANS in order to gain further insight into possible correlations between the film structure and the electrical conductivity of UNCD films.

This paper is organized as follows. Section II concerns the experimental details of the synthesis of the films and the different techniques employed to characterize and study them. In Section III, we present our main experimental observations and analysis. Section IV deals with the relationship between electrical conductivity and nitrogen incorporation, concentration, as well as bonding in UNCD. The structure of UNCD nanowires and the nature of $s p^{2}$ bonding in the carbon sheath surrounding the nanowires is discussed next. Finally, the current status of theories concerning electron transport in UNCD films is summarized. Conclusions constitute the final section (Sec. V) of the paper.

\section{METHODS}

Microwave plasma-enhanced chemical-vapor deposition (MPECVD) process has been used for the UNCD films synthesis. The depositions were carried out on both $\mathrm{Si}$ wafers and $\mathrm{Si}$ wafers with a thick thermal oxide using 6 in. CYRANNUS Innovative Plasma Systems (I PLAS GmbH) MPECVD system. The microwave power and the pressure were kept at $1200 \mathrm{~W}$ and 110 Torr, respectively. The substrate temperatures were varied in the range $750-940{ }^{\circ} \mathrm{C}$.

Argon-rich, nitrogen-rich $\mathrm{Ar} / \mathrm{CH}_{4} / \mathrm{N}_{2}$ plasmas were investigated for this study with a nominal gas composition of $1 \%$ for the $\mathrm{CH}_{4}$ and variable $\mathrm{Ar}$ and $\mathrm{N}_{2}$ flow rates so that the total gas flow was always 100 SCCM (SCCM denotes cubic centimeter per minute at STP). As nitrogen is added to the gas mixture, the relative partial pressure of argon was reduced, so that when $20 \% \mathrm{~N}_{2}$ is added, the exact composition is $1 \% \mathrm{CH}_{4}, 79 \% \mathrm{Ar}$, and $20 \% \mathrm{~N}_{2}$. The nitrogen content was varied in the range $5 \%-25 \%$.
The as-grown films were investigated using a HITACHI S-4700 field-emission gun (FEG) scanning electron microscope.

Raman spectra at excitation wavelength of $633 \mathrm{~nm}$ were collected at room temperature using a Renishaw microRaman RM2000 on a $50 \times$ objective. The spot size used was $\sim 1 \mu \mathrm{m}$.

The electrical properties of the samples have been assessed by four-point-probe resistivity measurements (Jandel Equipment). A high impedance current source is used to supply current through the outer two probes; a voltmeter measures the voltage across the inner two probes to determine the sample resistivity.

Transmission electron microscopy (TEM) investigations were performed on a Tecnai F20 FEG TEM/scanning TEM (STEM) microscope operating at an accelerating voltage of $200 \mathrm{keV}$. The point-to-point resolution of this microscope is $0.24 \mathrm{~nm}$. The high brightness FEG source allows the formation of small probes $(\sim 1 \mathrm{~nm})$ with an energy spread of $0.8 \mathrm{eV}$ (from the full width at half maximum of the zero loss peak). This provides appropriate conditions to record spectroscopic information at the nanometer scale from individual nanostructures. This microscope is equipped with a Gatan imaging filter for EELS acquisition. The electron-energy-loss (EEL) spectra were recorded in scanning TEM (STEM) mode, providing an incident beam with a convergence semiangle of $15 \mathrm{mrad}$ and a collection semiangle close to $7 \mathrm{mrad}$. This collection angle is much larger than the characteristic scattering angle of carbon in order to have a reasonable beam current into the spectrometer. The acquisition time was $8 \mathrm{~s}$ for each spectrum and the energy dispersion was $0.5 \mathrm{eV} / \mathrm{channel}$. The spectra were corrected for dark current and intrinsic channel-to-channel gain variations of the charge-coupled device (CCD), and the background below the ionization edges was subtracted by using the power-law model. ${ }^{15}$ The electron-diffraction patterns were acquired from sample regions about $150 \mathrm{~nm}$ in diameter on a CCD camera and with acquisition times of $4 \mathrm{~s}$. The TEM samples were prepared following standard routines: cutting, mechanical polishing, dimpling after a thickness of $\sim 15 \mu \mathrm{m}$, and milling with an argon-ion beam.

SANS experiments were carried out in the small-angle scattering instrument (SASI) at the Intense Pulsed Neutron Source at Argonne National Laboratory. SASI is a time-offlight SANS machine which covers a fairly wide $q$ range from 0.007 to $1.45 \AA^{-1}$ in a single experiment. ${ }^{20}$ SANS experiments in transmission were used to probe the average structure of the samples in a region of approximately $8 \mathrm{~mm}$ in diameter. One sample was tilted $45^{\circ}$ with respect to the beam direction to probe for anisotropy parallel to the wafer surface. A Si substrate was used as a blank to determine the background. The raw data were analyzed using standard procedures. $^{21}$

\section{RESULTS}

\section{A. Conductivity measurements}

UNCD films are insulators, which, when produced without $\mathrm{N}_{2}$ become semiconductors for modest $\mathrm{N}_{2}$ additions and 


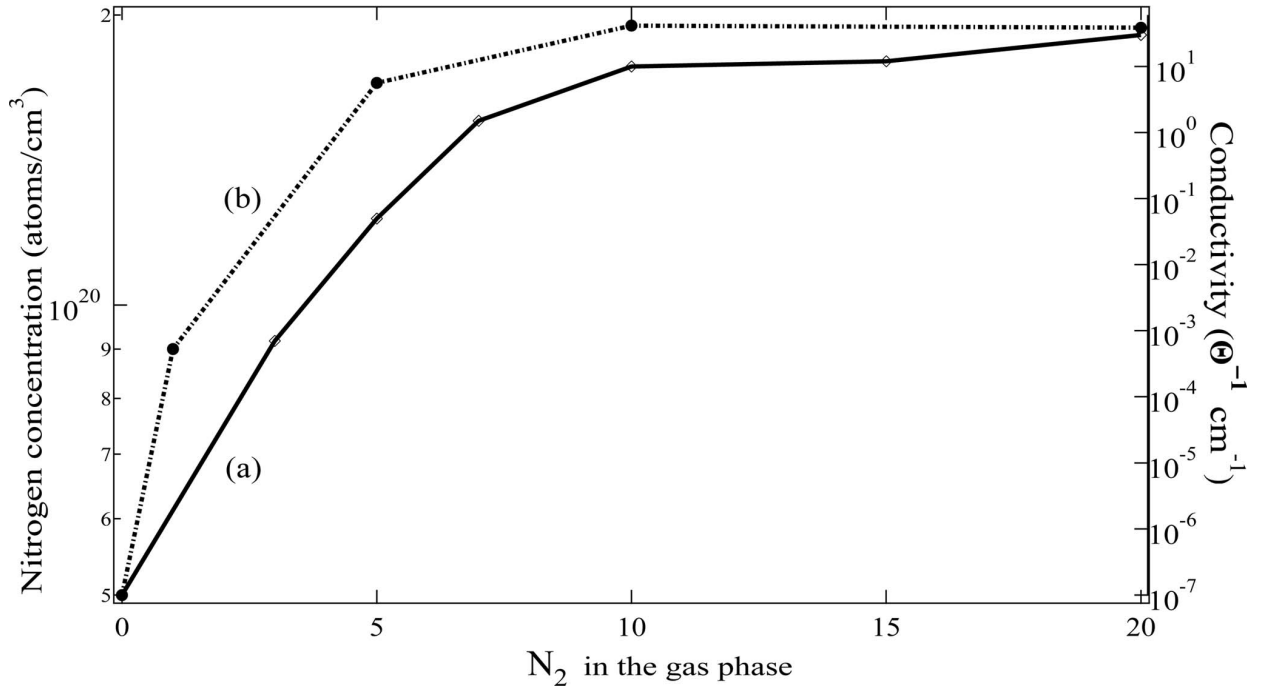

FIG. 1. (a) Conductivity and (b) secondary-ion-mass spectroscopy of UNCD films (Refs. 2) as a function of nitrogen concentration within the gas phase. finally display metal-like behavior at the highest $\mathrm{N}_{2}$ contents. This is evident by inspection of Fig. 1, where, in logarithmic scale, the conductivity, together with the number of nitrogen atoms $/ \mathrm{cm}^{3}$ incorporated in the grain boundaries (as determined by secondary-ion-mass spectrometry measurements ${ }^{22}$ ), is plotted as a function of nitrogen concentration within the gas phase.

\section{B. Scanning electron microscopy}

The abrupt change in conductivity behavior described above, reminiscent of a "phase" change, suggests a microstructural transformation that could account for the occurrence of the semiconductor-metal transition. Detailed SEM studies provide new insights concerning the underlying microstructural changes that help shed light on the complex conductivity behavior of $n$-type UNCD films.

UNCD films were deposited to thicknesses of $2-3 \mu \mathrm{m}$ on $\mathrm{Si}$ and $\mathrm{Si} / \mathrm{SiO}_{2}$ substrates in the temperature range between 800 and $950{ }^{\circ} \mathrm{C}$ and with $5 \%, 7 \%, 10 \%, 15 \%$, and $20 \%$ nitrogen substituting for Ar in the synthesis gas. After deposition, the Si wafers were cut to provide $1 \times 1 \mathrm{~cm}^{2}$ pieces for study in a scanning electron microscope.

Particular attention has been devoted to the influence of substrate temperature during the deposition as dictated by the voltage applied to the graphite susceptor placed under the

TABLE I. Processing conditions and conductivity values for samples studied in this work.

\begin{tabular}{lcc}
\hline \hline $\begin{array}{l}\text { Nitrogen } \\
(\%)\end{array}$ & $\begin{array}{r}\text { Temperature } \\
\left({ }^{\circ} \mathrm{C}\right)\end{array}$ & $\begin{array}{c}\text { Conductivity } \\
(\Omega \mathrm{cm})^{-1}\end{array}$ \\
\hline 5 & 800 & 0.14 \\
10 & 800 & 5 \\
15 & 800 & 25 \\
20 & 800 & 200 \\
10 & 800 & 5 \\
10 & 850 & 160 \\
10 & 940 & 110 \\
\hline \hline
\end{tabular}

substrate holder. Table I presents synthesis conditions for two series of samples together with the conductivity values as assessed by four-probe conductivity measurements. The first set of UNCD films was deposited at $800{ }^{\circ} \mathrm{C}$ with varying the nitrogen content in the range $0 \%-20 \%$. In the second set, the variation of the film properties as a function of substrate temperatures in the range between 800 and $940{ }^{\circ} \mathrm{C}$ for a fixed value of nitrogen addition to the gas phase (10\%) was studied.

An examination of Fig. 2(a) shows that even at 5\% nitrogen and $800{ }^{\circ} \mathrm{C}$, the randomly oriented $3-5 \mathrm{~nm}$ crystallites commonly observed in the absence of nitrogen already show a small degree of elongation which becomes more pronounced with continued increase in plasma nitrogen content. A drastic elongation manifests itself at $15 \%$ nitrogen content, which is perhaps best ascribed as due to the formation of diamond nanowires with aspect ratios of 20-30. They appear to be at least partially aligned in the plane. The addition of nitrogen to the gas phase at levels higher than $15 \%$ leads to the formation of highly dense and uniform distribution of finely structured diamond nanowires.

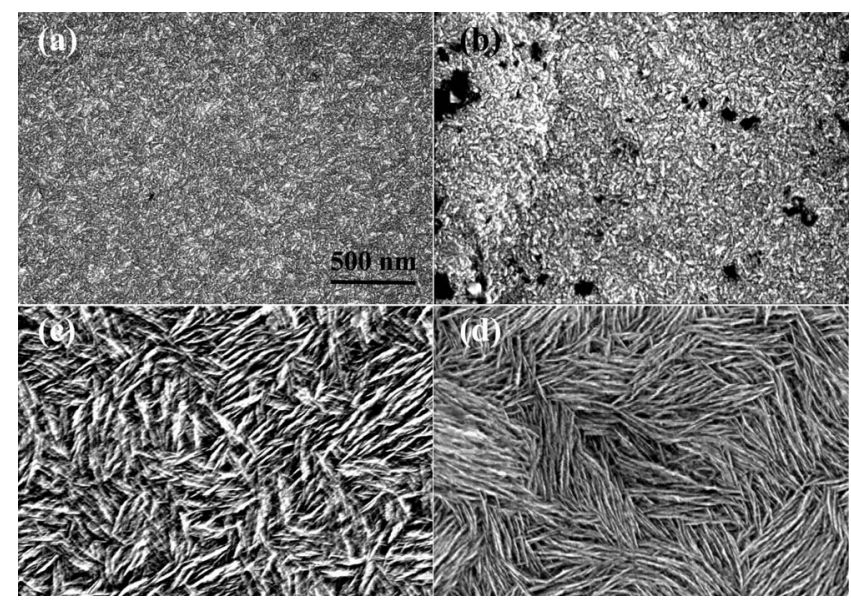

FIG. 2. Scanning electron microscopy showing morphology changes of the UNCD films as a function of nitrogen content [(a) $5 \%$, (b) $10 \%$, (c) $15 \%$, and (d) $20 \%$ ] at a substrate temperature of $800{ }^{\circ} \mathrm{C}$. The scale bar [in (a)] is the same for all the micrographs. 


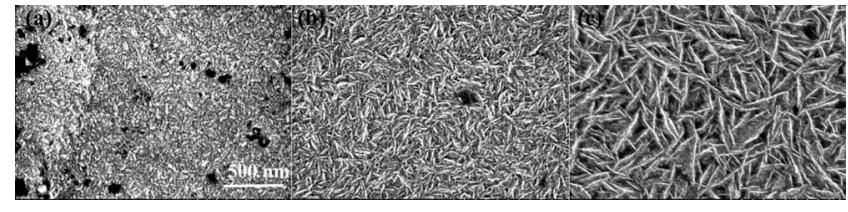

FIG. 3. Scanning electron microscopy showing the morphology variation of as-deposited UNCD nanofibers synthesized keeping the nitrogen content at $10 \%$ and the substrate temperature: (a) $800{ }^{\circ} \mathrm{C}$, (b) $850{ }^{\circ} \mathrm{C}$, and (c) $940{ }^{\circ} \mathrm{C}$. The scale bar [in (a)] is the same for all the micrographs.

Films obtained under essentially identical deposition conditions except that the deposition temperature was raised to $850{ }^{\circ} \mathrm{C}$ or higher show the formation of wires even at $10 \%$ nitrogen as clearly seen in Figs. 3(b) and 3(c). Growth of diamond nanowires is enhanced both by increased nitrogen content and/or by an increase in deposition temperature. At deposition temperatures below about $700{ }^{\circ} \mathrm{C}$, diamond nanowire formation ceases even in the presence of $20 \%$ nitrogen.

\section{Transmission electron microscopy: HRTEM, SAED, and EELS}

Further structural characterizations of the nanowires were performed with transmission electron microscopy. Figure 4(a) shows the low-magnification TEM micrograph corresponding to a general view of the samples. This image reveals the relatively homogeneous morphology of the samples with high-density nanoelongated wires randomly oriented in the plane. This random distribution of the wires indicates the absence of any pronounced texture in the plane of the film.

The wires have a uniform diameter of $\sim 5 \mathrm{~nm}$ along their entire length, which is between 80 and $100 \mathrm{~nm}$. HRTEM images of a typical wire are displayed in Figs. 4(b) and 4(c). These images show lattice fringes with a lattice spacing of $\sim 0.21 \mathrm{~nm}$, corresponding to the $d$ spacing of the (111) planes of the diamond. ${ }^{23}$ The image in Fig. 4(c) reveals a thin amorphous layer of order $1 \mathrm{~nm}$ in thickness that covers the surface of the diamond nanowires. This amorphous layer is likely formed during the growth of the wires. While it is difficult to measure a precise thickness for this amorphous layer due to delocalization effects in the electron microscope, ${ }^{24,25}$ the presence of this layer is confirmed by STEM-EELS (Fig. 6).

Figure 4(d) shows a HRTEM image of the matrix in which the nanowires are embedded. This matrix is composed of randomly oriented 3-5 $\mathrm{nm}$ crystallites of UNCD. The (111) lattice fringes of diamond planes are clearly visible in this micrograph. Figure 4(e) displays the SAED pattern recorded from the film (combination of the matrix and the nanowires). The SAED pattern demonstrates the existence of randomly oriented diamond crystallites and shows the large structural homogeneity and purity of the sample: sharp Bragg diffraction rings extending to $\{533\}$ are visible and no reflections attributed to graphite are observed. Scattering intensity from amorphous carbon was not detected, indicating that the amount of this material is very small.

Figure 5(a) shows that the structure of some of the nanowires is composed of several segments rather than a continuous structure. The size of these segments varies between 6 and $10 \mathrm{~nm}$. They exhibit atomically sharp interfaces, separated by structural defects, possibly twin boundaries, see Fig. 5 (b). The origin of this fragmentary structure is likely related to the growth mechanism of the wires. It is likely that this kind of structure is common but only visible under some imaging conditions.

Spatially resolved EELS was employed to identify the different structures present in the film at the nanometer scale. Figure 6 shows the carbon $K$-shell ionization edge EEL spectra recorded at different points in the film (size of the probe $\sim 1 \mathrm{~nm}$ ): (a) in the middle of one of the wires, (b) from the amorphous sheath at the edge of the wire, and (c) in the matrix. The fine structures of the $C-K$ edge of the EEL spectrum from one of these wires, shown in Fig. 4(a), present the

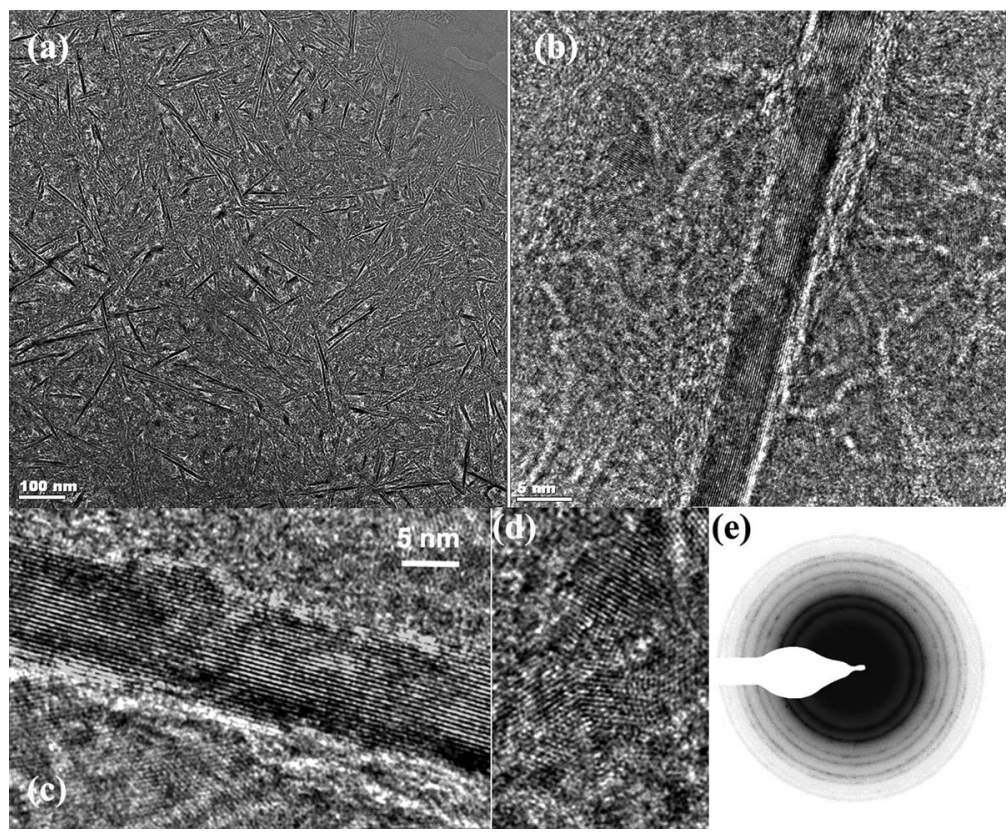

FIG. 4. (a) Low-magnification TEM image showing the mixture: matrix and UNCD nanowires. (b) High-magnification TEM micrograph displaying one of these nanowires. (c) HRTEM image to show the (111) diamond planes. (d) HRTEM image of one of the UNCD grains existing in the matrix [same scale bar as in (c)]. (e) Selected area diffraction pattern of the area including the matrix and the nanowires. 


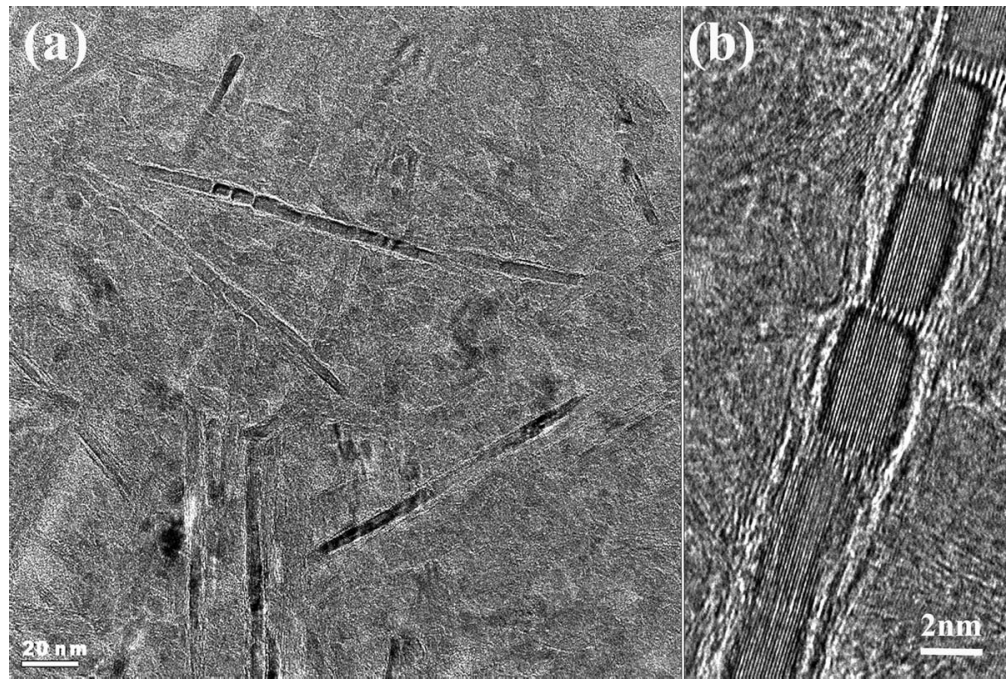

FIG. 5. (a) Low-magnification TEM image showing the UNCD nanowires. (b) HRTEM micrograph displaying the segments composing these nanowires. typical features of pure diamond involving $s p^{3}$ bonding configuration: ${ }^{26-28}$ (a) $\sim 289 \mathrm{eV}$ is the $\mathrm{C} 1 s$ core exciton resonance and (b) the large dip in the absorption spectrum at $\sim 302 \mathrm{eV}$ corresponds to the second absolute gap in the diamond band structure. ${ }^{26}$ Hence, EELS confirms unambiguously that these wires are diamond. Nevertheless, this EEL spectrum shows evidence of small fraction of $s p^{2}$ bonding: the relatively small pre-edge peak at $\sim 285 \mathrm{eV}$ attributed to $1 s \rightarrow \pi^{*}$ transitions in graphitelike $s p^{2}$-bonded carbon. ${ }^{27-29}$ This is also the case for the spectrum acquired in the matrix, Fig. 6(c), which is very similar to the spectrum recorded on the wire showing this preponderant $s p^{3}$ bonding hybridization. Contrary to these, the spectra acquired at the edge of the nanowire, e.g., Fig. 6(b), present $s p^{2}$ character. The presence of this amorphous layer is a key point, since it permits an explanation of the high conductivity of these samples compared to the values expected from the UNCD matrix or the diamond nanowires.

\section{Small-angle neutron scattering}

The deposited films are only few microns thick, but since UNCD films represent finely divided matter, there is suffi-

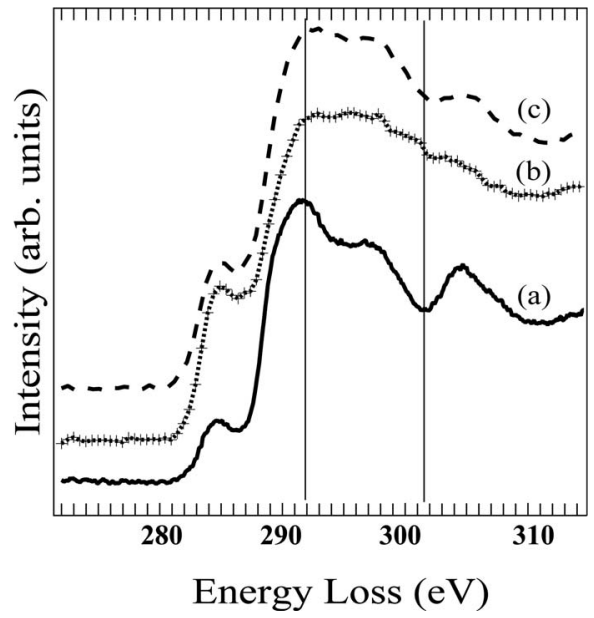

FIG. 6. EEL spectra showing the carbon $K$ edge obtained at different probe positions: (a) in the middle of a diamond nanowire, (b) at the edge of the nanowire, and (c) in the matrix. cient scattered intensity due to the large number of interfaces in the sample. The isotropic averaged neutron-scattering pattern shows that the morphology of the sample is different for all four samples (see Fig. 7). First, the scattering intensity increases with nitrogen content (even though the scattering intensity is normalized to approximate thicknesses of sample of $2 \pm 0.4 \mu \mathrm{m})$. The main feature of the data is the two distinct slopes. There is a slope change at approximately $2 \pi / 0.1 \AA^{-1} \sim 63 \AA$. A fit with a power law in the $q$-range 0.01-0.08 $\AA^{-1}$ in Fig. 7 shows an increasing exponent $(1.36 \rightarrow 1.93)$ with increasing plasma nitrogen concentration. This shows crossover from one-dimensional fiberlike structure with finite thickness to two-dimensional sheetlike structures measured above a length scale of $60 \AA$.

Figure 8 shows the SANS data as a Porod plot versus $q$. This representation of the data is a more sensitive way to check for the change in the slope of the data at approximately $0.15 \AA^{-1}$. This change in the slope is observed for all samples and is consistent with the size of UNCD crystallites (diameters of 3-5 $\mathrm{nm}$ ) reported in HRTEM images [Figs. 4(b) and 4(c)].

An examination of the sample with the highest nitrogen level with and without tilting the sample with respect to the beam shows a change in the scattering profile. This indicates that the sample is partly ordered (Fig. 9) on a nanometer length scale.

A comparison of the SANS measurements (Fig. 10 provides statistical information) with SEM images (Fig. 2 provides detailed local information) shows that the neutronscattering intensity is high in the samples where the SEM images show rodlike structures (see Fig. 10).

The measurements show that regardless of the small scattering volume of a UNCD film, meaningful information can be achieved with SANS measurements. However, the statistics is poorer at higher $q$-values due to the lack of intensity and also some of the structures on the films are too large to be resolved completely with a standard SANS instrument. The most surprising result from the SANS measurements is that neutrons see fibers in the low nitrogen concentration samples and higher dimension structures (sheetlike) for the two higher concentration samples. This seems to contradict the images from the SEM. This behavior could be interpreted 


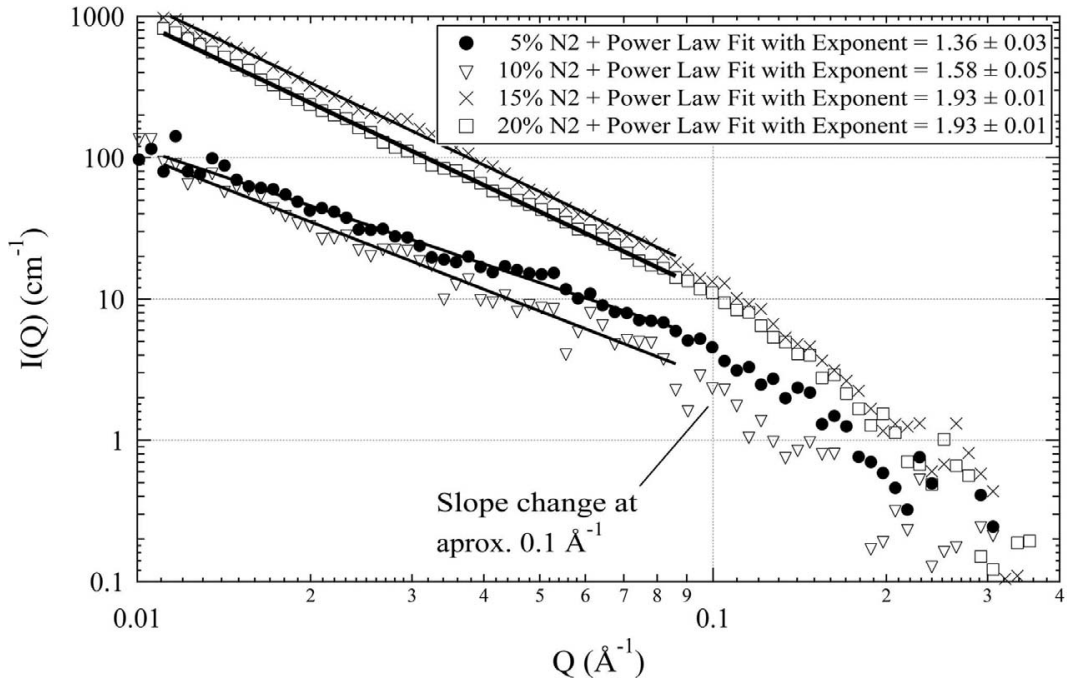

FIG. 7. Isotropic averaged neutron-scattering profile for the four film samples. in the way that the fibers are more ordered at higher concentration. Figure 9 supports ordering in the sample, since for a randomly oriented sample, the tilting should have increased the scattering volume and therefore the scattering intensity, but not the shape of the scattering profile. Further studies are underway to verify the degree of ordering in these films.

\section{DISCUSSION}

The remarkable electron transport, ${ }^{2-4}$ high-temperature diode, ${ }^{5}$ electrochemical, ${ }^{30}$ electron field emission, ${ }^{31}$ and optical properties ${ }^{32}$ of $n$-type UNCD films have been studied experimentally since their discovery about five years ago. Because of the many potential uses of diamond films with very high $n$-type conductivity (up to several hundred $\mathrm{S} / \mathrm{cm}$ even at ambient temperatures), the mechanism of electron transport has aroused considerable interest since their discovery.

The electrical conductivity of UNCD films is strongly correlated with the amount of incorporated nitrogen as shown in Fig. 1. A similar trend has been observed in a detailed optical emission spectroscopy study of the plasmas used for the UNCD film deposition for the variation of $\mathrm{CN} / \mathrm{C}_{2}$ ratios as a function of nitrogen content. ${ }^{33}$ Both the $\mathrm{CN} / \mathrm{C}_{2}$ ratios and the number of nitrogen atoms $/ \mathrm{cm}^{3}$ incorporated in the grain boundaries increase strongly in the region $0 \%-5 \%$ by volume of added nitrogen and then level off in the region $5 \%-20 \%$ added nitrogen. In the region where both $\mathrm{CN} / \mathrm{C}_{2}$ ratios and grain-boundary nitrogen contents increase strongly, changes in conductivity spanning many orders of magnitude occur. This enables the controlled variation of synthesis conditions so as to produce ambient conducting $n$-type diamond films showing the entire range from insulating to semiconducting behavior. On the reasonable supposition that the conductivity tracks an increasing nitrogen concentration in the grain boundaries, which, in turn, strongly increases the carrier density by providing a source of unpaired electrons, electron paramagnetic resonance was used to follow the postulated progression. However, the measurements, which also included spin-spin and spin-lattice relaxation data, failed to disclose a dependence of the spin density on nitrogen concentration leading to the

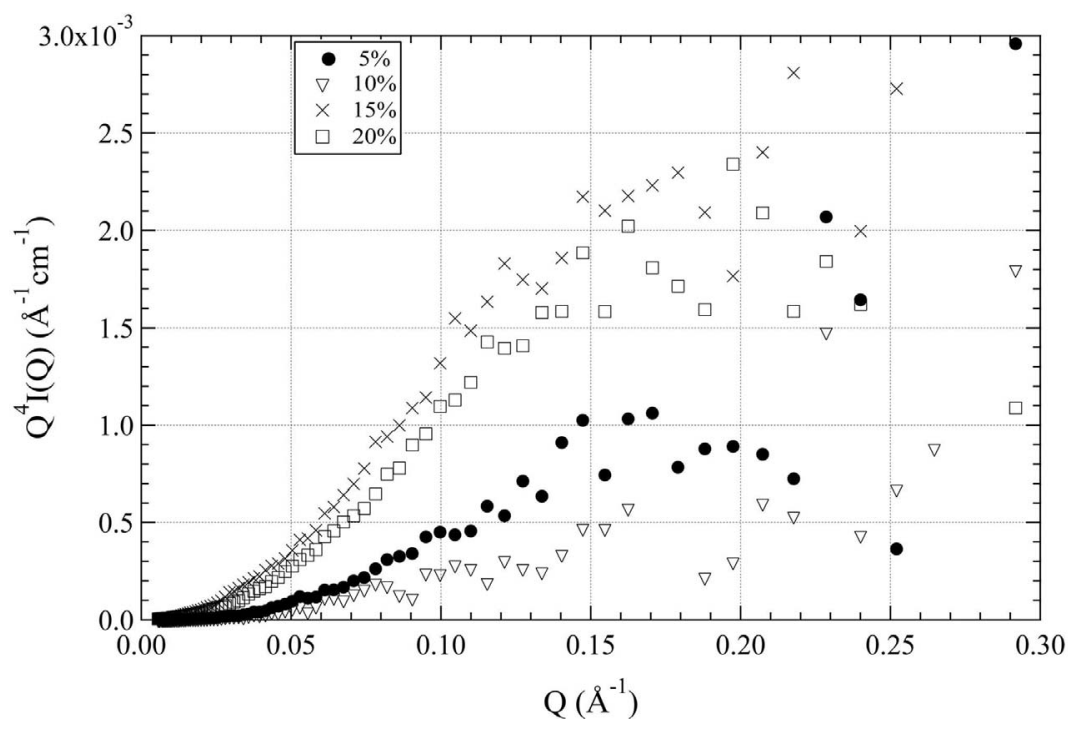

FIG. 8. Porod plot of the SANS data. 

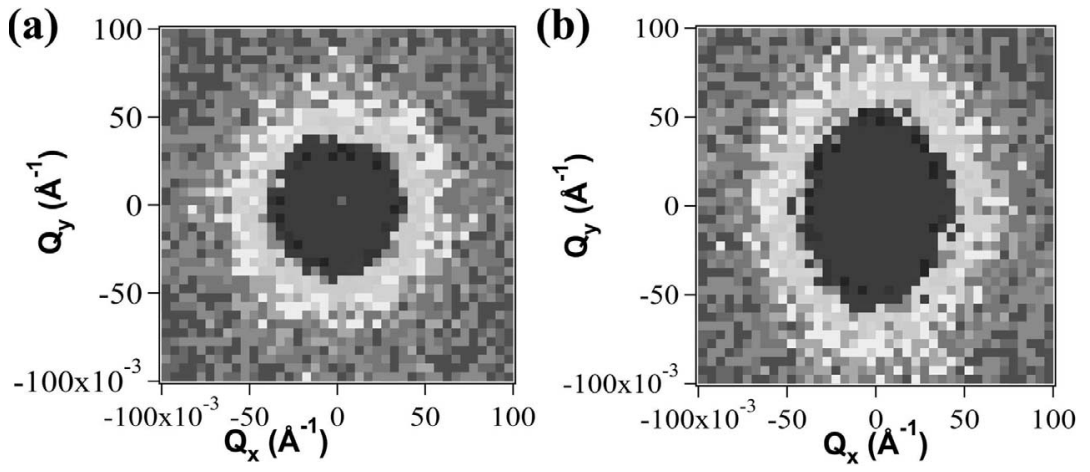

FIG. 9. Two-dimensional scattering image of the $20 \%$ nitrogen concentration sample measured with the neutron beam tilted (a) $90^{\circ}$ and (b) $45^{\circ}$ with respect to the UNCD film.

conclusion that the proposed mechanism cannot, in fact, explain the large conductivity changes. ${ }^{34}$

In the "leveling off" region, the conductivity continues to increase by several orders of magnitude with increasing nitrogen content. Moreover, the conductivity changes are accompanied by a changeover from strong to weak temperature dependence, suggesting that a transition from semiconducting hopping conductivity $(0 \%-5 \%$ nitrogen) to metallic conductivity (5\%-20\% nitrogen) occurs in this regime. Surprisingly, the transition takes place over a variation of plasma nitrogen content of only a few percent.

Since lattice substitutional nitrogen, which occupies a level situated $1.7 \mathrm{eV}$ below the conduction band, cannot contribute to the conductivity in the temperature range of interest here, a mechanism not involving transport via the lattice must be invoked. This situation is in contradiction to phosphorus-doped diamond films, for example, where $n$-type electrical conductivity has been observed at high temperatures. ${ }^{35,36}$ In those materials, the conductivity is plausibly due to lattice mediated transport, since phosphorus occupies substitutional sites that lie only $0.6 \mathrm{eV}$ below the dia- mond conduction band. It is of interest briefly to review the key features of an alternative transport mechanism involving the grain boundaries that take the place of the lattice in UNCD to provide $n$-type conductivity even at ambient temperatures. The ideas discussed here are based on detailed theoretical considerations and on extensive grain-boundary bonding, as well as morphological studies culminating in the present work which shows that the formation of nanowires with diamond cores leads to partially aligned nanostructures with highly effective electron-transport pathways.

Approximately $10 \%$ of all carbon atoms in UNCD films prepared without $\mathrm{N}_{2}$ added participate in bonding at the grain boundaries (GBs). Keblinski et al., using moleculardynamics simulations, showed that the carbon bonding at the $0.2 \mathrm{~nm}$ wide high-angle high-energy twist grain boundaries is very complex consisting of $s p^{2}, s p^{2+x}$, and $s p^{3}$ hybridizations and that as a consequence the band gap of diamond is not empty but filled with partially delocalized electronic states. $^{7,8}$ The $0.2 \mathrm{~nm}$ width of GBs in nonnitrogen containing films has been confirmed experimentally by HRTEM studies. ${ }^{10}$
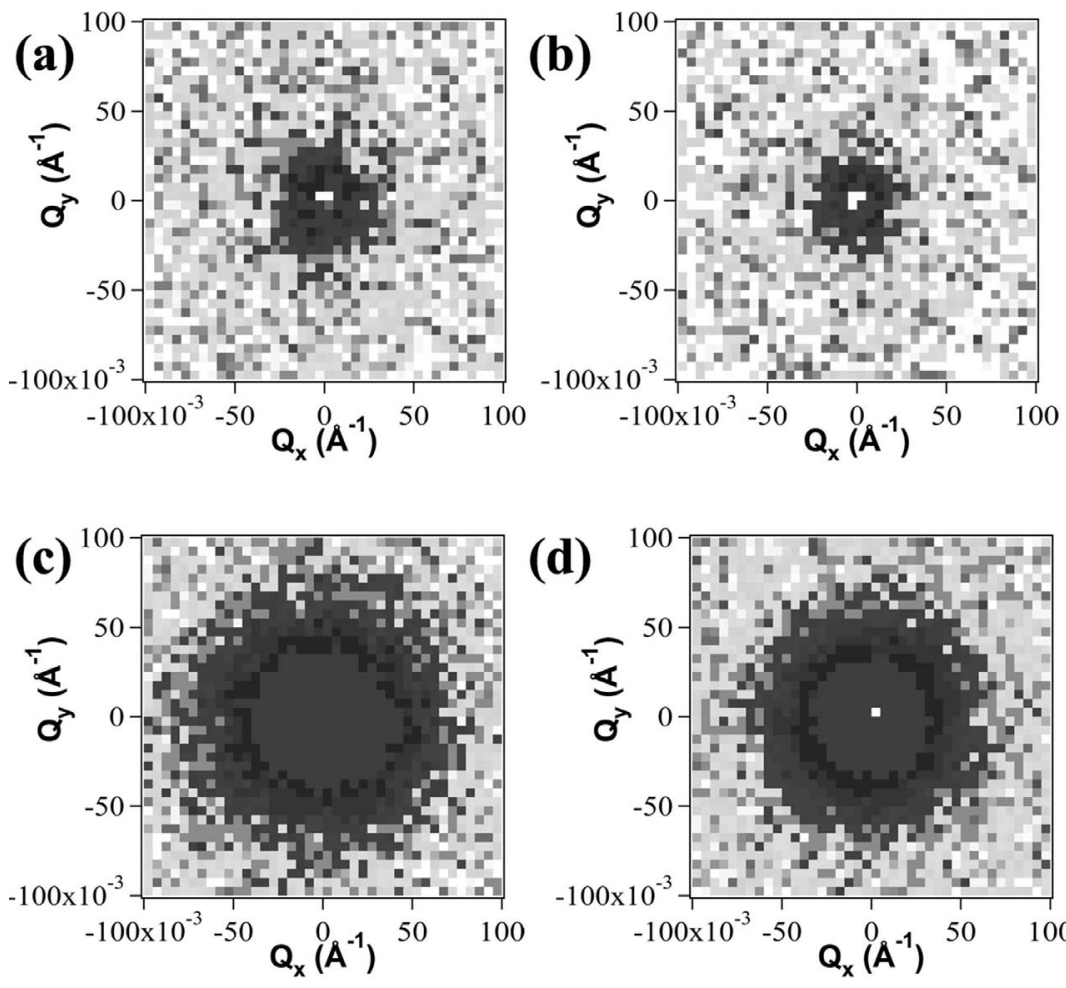

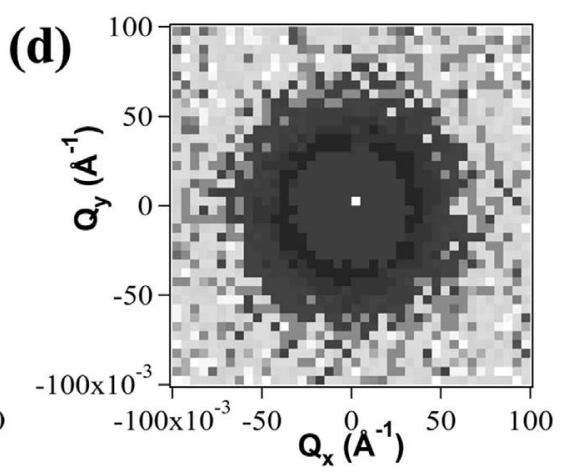

FIG. 10. SANS profiles obtained from four different samples corresponding to SEM images [Fig. 2(a)-2(d)]. 
The dramatic changes in conductivity that accompany the incorporation of nitrogen into UNCD films were evaluated by density-functional-based tight-binding (DFTB) calculations that revealed different possible nitrogen bonding configurations. ${ }^{9}$ The most stable configuration in the grain boundary turns out to have a formation energy of $-0.64 \mathrm{eV}$ compared to a substitutional site in the diamond crystal lattice of $4.9 \mathrm{eV}$. This large energy difference essentially dictates that nitrogen incorporation occurs by substitution into the grain boundaries. Nitrogen lone pairs hybridized with carbon dangling bond states are situated above the Fermi level and can donate electrons to carbon defect states near the Fermi level causing it to shift upward toward a delocalized $\pi^{*}$ band. Increasing the nitrogen content leads to additional changes in electronic structure, which have, as a consequence, an increase of $s p^{2}$-bonded carbon as well as still higher delocalization and broadening of the $\pi^{*}$ band. Similar conclusions have been reached in more recent work using density-functional-theory (DFT) calculations based on specific nitrogen containing carbon cluster models. ${ }^{37}$

The many orders of magnitude changes in electrical conductivity due to the addition of nitrogen to the synthesis gas are due not only to the changes in the electronic structure of energy levels introduced into the band gap of diamond that have just been described but also to the interplay of these with UNCD film nanostructural and bonding changes. The latter have been studied by a variety of techniques including HRTEM, EELS, near-edge x-ray-absorption fine structure, Raman spectroscopy, and x-ray diffraction (XRD). ${ }^{11-13}$ There appears to be a general agreement, as a result of this work, that the width of the grain boundaries gradually increases with increasing nitrogen content from 0.2 to between 1 and $2 \mathrm{~nm}$. The grain-boundary carbon displays a pronounced degree of $s p^{2}$ bonding in agreement with the DFTB calculations discussed above. There is a disagreement, however, concerning the effect of nitrogen additions on the crystallite size. HRTEM results have been interpreted in terms of increasing grain size. XRD measurements, on the other hand, reveal no increase in crystallite size. They do show an increase in the $(111) /(220)$ intensity ratio from 3.5 to 5.0 in going from $0 \%$ to $20 \%$ nitrogen, indicating an enhancement of (111) orientation. Thus, a key question that could help elucidate the mechanism of grain-boundary conductivity still remains in spite of the extensive work that has already been done. It concerns the fraction of $s p^{2}$-bonded carbon in the films and the structural changes that on occasion suddenly increase in $s p^{2}$-bonded carbon connectivity particularly during the transition to metallic behavior. To help answer this question, the present detailed re-examination of UNCD nanostructural changes as a result of nitrogen additions was undertaken.

As pointed out in Sec. III B, highly elongated diamond crystallites with aspect ratios of 20-30 gradually replace the $3-5 \mathrm{~nm}$ randomly oriented crystallites typical of nonnitrogen containing films. They become particularly pronounced when nitrogen contents of $10 \%$ and above are combined with deposition temperatures of about $800{ }^{\circ} \mathrm{C}$ and above [see Figs. 2(b)-2(d)]. Films displaying metallic conductivity behavior are found always to possess the characteristic features of these diamond rods which we call nanowires, thus dem-

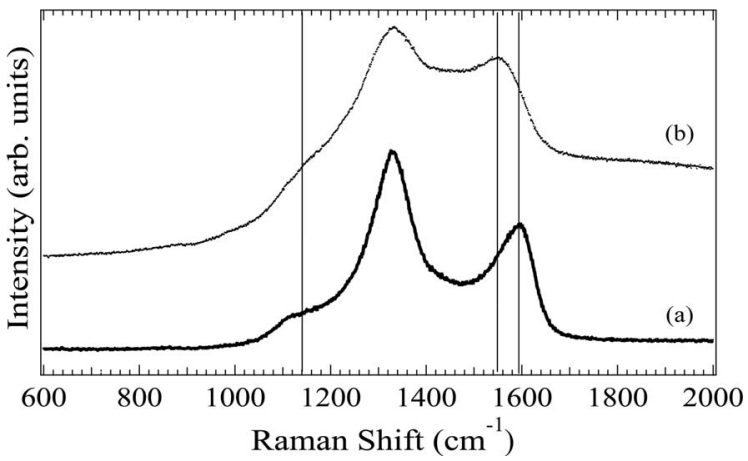

FIG. 11. Raman spectra for two different UNCD samples: (a) $20 \%$ and (b) $0 \%$ of nitrogen added to the synthesis gas (substrate temperature of $800{ }^{\circ} \mathrm{C}$ during deposition).

onstrating the close relationship between conductivity and the nanowire film structure. A typical Raman spectrum of a film deposited at $800{ }^{\circ} \mathrm{C}$ in the presence of $20 \%$ nitrogen [Fig. 11(a)] and possessing the nanowire structure is compared to a film prepared in the absence of nitrogen [Fig. 11(b)]. The principal differences in the two spectra are the shift of the $G$ peak from 1546 to $1590 \mathrm{~cm}^{-1}$, a small increase in the $I(D) / I(G)$ ratio, and a decrease in the scattering intensity for the $n$-type film. These changes are consistent with an increase in $s p^{2}$ bonding character of the grain-boundary carbon due to the incorporation of nitrogen. Similar interpretations of Raman spectra on $n$-type UNCD films have been given by Vlasov et al. ${ }^{38}$ In films prepared using $10 \%$ added nitrogen, these workers, using surface-enhanced Raman scattering, also found evidence for carbodiimide and paracyanogenlike chains as well as stretching modes of nitrile or cyanamide groups conjugated to aromatic rings. It is likely that such chainlike structures consisting of $s p^{2}$ and $s p^{1}$ hybridized $\mathrm{C}$ and $\mathrm{N}$ can, in concert with aromatic carbon ring clusters, form a rigid connective network leading to highly delocalized electronic states which translate not only into increased electrical conductivity but also into an increase in optical absorption due to a decrease in the optical band gap. 32,39-41

The HRTEM studies show that the 80-100 nm long nanowires are composed of an $s p^{3}$-bonded diamond core consisting of $5 \mathrm{~nm}$ wide, 6-10 nm long segments exhibiting atomically sharp interfaces probably separated by twin boundaries. Each nanowire is enveloped in an amorphous sheath. The sheath is composed of largely $s p^{2}$-bonded carbon as determined by EELS measurements. Determination of the growth direction requires further XRD and, in particular, pole figure data. Perhaps, as is frequently the case with CVD diamond, (110) may be the direction of the fastest growth here as well. In any event, the "selective evolution model" that has been postulated for ballaslike growth of $5 \mathrm{~nm}$ diamond crystallites and that favors the (110) direction for each crystallite in the absence of nitrogen incorporation ${ }^{42}$ clearly requires modification under the conditions favoring nanowire formation considered here. The mechanism responsible for the linking up of 10-20 crystallite segments to form a nanowire poses an intriguing problem. Arguments for an interactive process involving both the diamond and the $s p^{2}$-bonded 
carbon growing simultaneously in the plasma environment are presented in the following.

The proposal that the principal UNCD growth species is carbon dimer, $\mathrm{C}_{2}$, produced in microwave plasmas by fragmentation of acetylene, has found substantial experimental as well as theoretical support. ${ }^{1}$ The role of the precursor of $\mathrm{C}_{2}$, acetylene, in the diamond growth process has also been elucidated. It has been concluded that the grain boundaries surrounding UNCD crystallites in nonnitrogen containing films are composed of trans-polyacetylene-like $s p^{2}$-bonded carbon. ${ }^{38}$ The evidence that this is the case comes primarily from Raman data which show, for example, a peak at about $1140 \mathrm{~cm}^{-1}$ (see Fig. 11). This feature coincides with both the carbon-carbon single bond stretching and the carbonhydrogen bond in-plane bending modes of trans-polyacetylene. ${ }^{14,43,44}$ The chemistry of the synthesis gas becomes considerably more complex with the addition of nitrogen. Species other than $\mathrm{C}_{2} \mathrm{H}_{2}$ that lead to the formation of polyacetylene must then be considered. CHEMKIN calculations show that at $1000 \mathrm{~K}$, when nitrogen is present in addition to methane and argon, $\mathrm{HCN}$ becomes the dominant species particularly with increasing neutral gas temperatures. ${ }^{33}$

Polyacetylene is, of course, well known, but in actual fact, polymeric $\mathrm{HCN}$ was the first synthetic organic polymer ever to be discovered. ${ }^{45,46}$ It is thermally unstable and decomposes into a copolymer of polymethineimine (polynitrile) and polyacetylene with the liberation of nitrogen.$^{47}$ The formation of a copolymer during UNCD synthesis is likely since the microwave plasma strongly enhances polymerization reactions. Excitation processes due to collisions with $\mathrm{Ar}^{+}$and $\mathrm{Ar}^{*}$ (Ref. 48) lead to the formation of a complex mixture of organic molecules including trans-polyacetylene and polycyclic aromatic hydrocarbons even in the absence of added nitrogen. ${ }^{41}$ The formation of UNCD grain boundaries provisionally described as partaking of the properties of a copolymer of polyacetylene and polynitrile would go a long way in providing a basis for a plausible mechanism leading to the formation of diamond nanowires as well as rationalizing their extraordinary electrical and optical properties.

There is a very good match in the periodicity vector between polyacetylene and polynitrile (approximately $2.5 \AA$ ) with the unit-cell periodicities of the (100) and (111) surfaces of diamond (2.51 and $5.3 \AA$, respectively), suggesting that covalent bonding between the growing polymer chains and the diamond lattices can occur with little or no activation energy. The $\mathrm{C}-\mathrm{N}$ bond is about $20 \%$ stronger than the $\mathrm{C}-\mathrm{C}$ bond, providing for an increased stiffness of a polynitrile chain containing $\mathrm{C}-\mathrm{N}$ groups compared to a polyacetylene chain that does not. The copolymer chain would therefore tend to resist bending, thus presenting a suitable template for growing straight chains of $80-100 \mathrm{~nm}$ long diamond nanowires surrounded by a polymerlike sheath.

The increase in optical absorption and lowering of the band gap as nitrogen concentration increases in UNCD films reflect to some extent the nonlinear optical (NLO) properties of polynitrile/polyacetylene copolymers that possess very large hyperpolarizabilities. ${ }^{49}$ They have been the subject of extensive experimental and theoretical investigations because of their remarkable NLO and electrical conductivity characteristics. ${ }^{50}$ It is of interest to point out here that the values of conductivity, carrier density, and carrier mobility of $n$-type UNCD films in the metallic state are very close to those of polyacetylene itself when the polymer is heavily doped with $\mathrm{AsF}_{5} \cdot{ }^{51}$ Unlike polyacetylene, the UNCD films resist corrosion to an extraordinary degree and they are of course extremely temperature stable.

Electrical conduction through the $n$-type UNCD grain boundaries appears to be similar to that of conducting polymers even if these grain boundaries are compositionally and structurally very different from conventional polymers. To improve our knowledge of this point, further investigations at the nanometer scale are needed. TEM is one of the more appropriate techniques for such studies. However, it should be mentioned that the precise characterization of such materials is difficult due to the damage induced by the electron beam and the reduced size of such objects.

It is not possible on the basis of either the SEM or the HRTEM studies to determine whether or not the nanowires display ordering. In order to obtain such information, smallangle neutron-scattering (SANS) measurements were performed. Four films with varying nitrogen concentrations were used, and the results showed that the scattering intensity increases with nitrogen. Further data analysis reveals the existence of a crossover from a one-dimensional rodlike structure to two-dimensional sheetlike structures. For samples prepared by adding $20 \%$ nitrogen to the plasma, a change in the scattering profile was observed when the film was tilted $45^{\circ}$ with respect to the neutron beam. Partial ordering on nanometer length scale in planes parallel to the growth direction of the film can account for this phenomenon. One can therefore conclude, on the basis of the SANS studies, that the nanowires appear to be at least partially aligned parallel to the plane of the substrate. The transition to the metallic state therefore involves an enhancement of the connectivity due both to the formation of nanowires as well as to their partial alignment relative to each other.

Various theoretical considerations have been applied to an elucidation of electronic transport in $n$-type UNCD films. The application of DFTB theory to this problem has given considerable insight into the changes in the electronic structure of the band gap of diamond due to the incorporation of nitrogen. The finding that grain-boundary nitrogen enhances $\pi$ bonding and electron delocalization is particularly relevant to electron-transport mechanisms. ${ }^{9}$ In part, based on these ideas, conductivity mechanisms involving nitrogen-vacancy complexes as shallow donors and grain-boundary nitrogendangling bond or nitrogen- $\pi$ bond complexes as compensation centers have been proposed based on DFTB cluster calculations. ${ }^{37}$ Another approach, using a combination of hopping and delocalized state conduction for low and high nitrogen content UNCD, respectively, comes to the conclusion that the experimental data are in accord with the DFTB predictions that the Fermi level shifts toward the conduction band with increasing nitrogen incorporation. ${ }^{52}$ Recently, giant negative magnetoresistance has been found in $n$-type UNCD at very low temperatures, which was interpreted on the basis of grain-boundary conduction having the character of a low-dimensional disordered metal in weakly localized orbits that extend over the entire crystallite. ${ }^{39,40}$ A consensus appears to be emerging that the insulator-metal transition in 
$n$-type UNCD involves a changeover from variable range hopping (VRH) transport in localized states near the Fermi level to defect band conduction. ${ }^{3}$ However, the assumption of a constant density of states near the Fermi level inherent in Mott's VRH model leads to unphysical values of UNCD conductivity parameters such as the density of states, the localization length, and the average hopping distance. Following Godet, ${ }^{53}$ one assumes instead an exponentially varying density of states above the Fermi level as well as a localization length parameter that can vary, for example, with band tailing due to electronic disorder. The existence of a close relationship between electronic disorder and increasing localization length linked to an increase in grain-boundary width is not unexpected. Reasonable values for the densities of states at the Fermi level as well as for mobilities over the whole range of nitrogen concentrations are obtained if one increases the localization length from 5 to $20 \AA$. Using this increased localization length yields qualitative agreement with measured conductivities and activation energies. ${ }^{3}$ The crossover region from the insulator to the metal regime can be said to occur when the screening length and the localization length approximately equal one another. An expression was obtained that relates the critical concentration of carriers needed for metallic conduction to the inverse third power of the localization length. ${ }^{54}$ In this treatment, one assumes, importantly, that the localization length can, in fact, be approximated by the grain-boundary width, which, as described above, has been found experimentally to increase with increasing nitrogen incorporation. For a grain-boundary width of $1 \mathrm{~nm}$, typical of HRTEM results on diamond nanowires presented in this paper, one calculates the critical concentration of carriers to be in the range $\left(10^{19}-10^{21}\right) / \mathrm{cm}^{3}$ in accord with results from Hall measurements. ${ }^{4}$ That the influence of a profound increase in connectivity is likely associated with the morphology change of the film due to the appearance of nanowires has not yet been incorporated into theoretical considerations concerning metallic conductivity in $n$-type UNCD. Thus, this point needs further theoretical developments.

\section{CONCLUSION}

In summary, we studied the modifications in structure and properties of UNCD films as a function of the addition of nitrogen gas during their synthesis. We concluded that the drastic changes of the electrical conductivity behavior of these films when the nitrogen-argon ratios are increased are strongly correlated to significant changes in microstructure of the UNCD films. In particular, we showed the transformation from randomly oriented 3-5 $\mathrm{nm}$ diamond crystallites to diamond nanowires surrounded by a largely $s p^{2}$-bonded carbon sheath when $\mathrm{N}_{2}$ contents of $10 \%$ and above are combined with deposition temperatures of about $800^{\circ} \mathrm{C}$ and above. We suggest that this sheath, of order $1 \mathrm{~nm}$ in thickness, is responsible for the high conductivity of these UNCD films. Partial alignment of the nanowires is evidenced by small-angle neutron-scattering (SANS) investigations.

\section{ACKNOWLEDGMENTS}

This work was supported by the U.S. Department of Energy, Office of Science, under Contract No. DE-AC0206CH11357. The electron microscopy work (SEM and TEM) was carried out in the Electron Microscopy Center at Argonne National Laboratory, which is also supported by the Office of Science.
${ }^{1}$ D. M. Gruen, Annu. Rev. Mater. Sci. 29, 211 (1999).

${ }^{2}$ S. Bhattacharyya, O. Auciello, J. Birrell, J. A. Carlisle, L. A. Curtiss, A. N. Goyette, D. M. Gruen, A. R. Krauss, J. Schlueter, A. Sumant, and P. Zapol, Appl. Phys. Lett. 79, 1441 (2001).

${ }^{3}$ P. Achatz, O. A. Williams, P. Bruno, D. Gruen, J. A. Garrido, and M. Stutzmann, Phys. Rev. B 74, 155429 (2006).

${ }^{4}$ O. A. Williams, S. Curat, J. E. Gerbi, D. M. Gruen, and R. B. Jackman, Appl. Phys. Lett. 85, 1680 (2004).

${ }^{5}$ T. Zimmermann, M. Kubovic, A. Denisenko, K. Janischowsky, O. A. Williams, D. M. Gruen, and E. Kohn, Diamond Relat. Mater. 14, 416 (2005).

${ }^{6}$ O. A. Williams, Semicond. Sci. Technol. 21, R49 (2006).

${ }^{7}$ P. Keblinski, D. Wolf, S. R. Phillpot, and H. Gleiter, J. Mater. Res. 13, 2077 (1998).

${ }^{8}$ F. Cleri, P. Keblinski, L. Colombo, D. Wolf, and S. R. Phillpot, Europhys. Lett. 46, 671 (1999).

${ }^{9}$ P. Zapol, M. Sternberg, L. A. Curtiss, T. Frauenheim, and D. M. Gruen, Phys. Rev. B 65, 045403 (2002).

${ }^{10}$ S. Jiao, A. Sumant, M. A. Kirk, D. M. Gruen, A. R. Krauss, and O. Auciello, J. Appl. Phys. 90, 118 (2001).

${ }^{11}$ J. Birrell, J. A. Carlisle, O. Auciello, D. M. Gruen, and J. M. Gibson, Appl. Phys. Lett. 81, 2235 (2002).

${ }^{12}$ J. E. Gerbi, I. Auciello, J. M. Gibson, D. M. Gruen, and J. A.
Carlisle, J. Appl. Phys. 93, 5606 (2003).

${ }^{13}$ J. E. Gerbi, J. Birrell, M. Sardela, and J. A. Carlisle, Thin Solid Films 473, 41 (2005).

${ }^{14}$ T. Lopez-Rios, E. Sandre, S. Leclerq, and E. Sauvain, Phys. Rev. Lett. 76, 4935 (1996).

${ }^{15}$ R. F. Egerton, Electron Energy-Loss Spectroscopy in the Electron Microscope, 2nd ed. (Plenum, New York, 1996).

${ }^{16}$ R. Arenal, O. Stephan, M. Kociak, D. Taverna, C. Colliex, A. Rubio, and A. Loiseau, in Electric Properties of Synthetic Nanostructures, edited by H. Kuzmany, J. Fink, M. Mehring, and S. Roth, AIP Conf. Proc. No. 723 (AIP, New York, 2004), p. 293.

${ }^{17}$ R. Arenal, M. Kociak, and N. J. Zaluzec, Microsc. Microanal. 12, 1188 (2006).

${ }^{18}$ A. C. Ferrari, A. Libassi, B. K. Tanner, V. Stolojan, J. Yuan, L. M. Brown, S. E. Rodil, B. Kleinsorge, and J. Robertson, Phys. Rev. B 62, 11089 (2000).

${ }^{19}$ J. S. Higgins and H. C. Benoit, Polymers and Neutron Scattering, Oxford Series on Neutron Scattering in Condensed Matter Vol. 8 (Clarendon, Oxford, 1994).

${ }^{20} \mathrm{http} / / /$ www.pns.anl.gov/instruments/sasi

${ }^{21}$ P. Thiyagarajan, J. E. Epperson, R. K. Crawford, J. M. Carpenter, T. E. Klippert, and D. G. Wozniak, J. Appl. Crystallogr. 30, 280 (1997). 
${ }^{22}$ These secondary-ion-mass spectroscopy data are obtained from an earlier work (Ref. 2).

${ }^{23}$ This is in good agreement with the value reported $(F d \overline{3} m, a$ $=0.357 \mathrm{~nm})($ Ref. 1).

${ }^{24}$ W. Coene and A. J. E. M. Jansen, Scanning Microsc. Suppl. 6, 379 (1992).

${ }^{25}$ H. W. Zandbergen, D. Tang, and D. Van Dyck, Ultramicroscopy 64, 185 (1996).

${ }^{26}$ J. F. Morar, F. J. Himpsel, G. Hollinger, G. Hughes, and J. L. Jordan, Phys. Rev. Lett. 54, 1960 (1985).

${ }^{27}$ H. K. Schmid, Microsc. Microanal. Microstruct. 6, 99 (1995).

${ }^{28}$ K. Okada, K. Kimoto, S. Komatsu, and S. Matsumoto, J. Appl. Phys. 93, 3120 (2003).

${ }^{29}$ A. J. Papworth, C. J. Kiely, A. P. Burden, S. R. P. Silva, and G. A. J. Amaratunga, Phys. Rev. B 62, 12628 (2000).

${ }^{30}$ Q. Chen, D. M. Gruen, A. R. Krauss, T. D. Corrigan, M. Witek, and G. M. Swain, J. Electrochem. Soc. 148, E44 (2001).

${ }^{31}$ T. D. Corrigan, D. M. Gruen, A. R. Krauss, P. Zapol, and R. P. H. Chang, Diamond Relat. Mater. 11, 43 (2002).

${ }^{32}$ P. Achatz, J. A. Garrido, M. Stutzmann, O. A. Williams, D. M. Gruen, A. Kromka, and D. Steinmuller, Appl. Phys. Lett. 88, 101908 (2006).

${ }^{33}$ D. M. Gruen and P. Bruno (unpublished).

${ }^{34}$ M. Rovere, S. Porro, A. Shames, O. Williams, S. Russo, P. Bruno, A. Tagliaferro, and D. M. Gruen, Diamond Relat. Mater. 15, 1913 (2006).

${ }^{35}$ S. Koizumi, K. Watanabe, M. Hasegawa, and H. Kanda, Science 292, 1899 (2001).

${ }^{36}$ M. Nesladek, Semicond. Sci. Technol. 20, R19 (2005).

${ }^{37}$ Y. Dai, D. Dai, C. Yan, B. Huang, and S. Han, Phys. Rev. B 71, 075421 (2005).

${ }^{38}$ I. I. Vlasov, V. G. Ralchenko, E. Goovaerts, A. V. Saveliev, and
M. V. Kanzyuba, Phys. Status Solidi A 203, 3028 (2006).

${ }^{39}$ J. J. Mares, P. Hubic, J. Kristofik, D. Kindl, M. Fanta, M. Nesladek, O. Williams, and D. M. Gruen, Appl. Phys. Lett. 88, 092107 (2006).

${ }^{40}$ M. Nesladek, D. Tromson, P. Bergonzo, P. Hubik, J. J. Mares, J. Kristofik, D. Kindl, O. A. Williams, and D. Gruen, Diamond Relat. Mater. 15, 607 (2006).

${ }^{41}$ N. Aggadi, C. Arnas, F. Benedic, C. Dominique, X. Duten, F. Silva, K. Hassouni, and D. M. Gruen, Diamond Relat. Mater. 15, 908 (2006).

${ }^{42}$ F. Silva, F. Benedic, P. Bruno, and A. Gicquel, Diamond Relat. Mater. 14, 398 (2005).

${ }^{43}$ A. C. Ferrari and J. Robertson, Phys. Rev. B 64, 075414 (2001).

${ }^{44}$ H. Kuzmany, R. Pfeiffer, N. Salk, and B. Gunther, Carbon 42, 911 (2004).

${ }^{45}$ J. L. Proust, Gehlen's J. Chem. Physik 3, 384 (1807); Annal. Chim. 60, 233 (1806).

${ }^{46}$ TH. Volker, Angew. Chem. 72, 379 (1960).

${ }^{47}$ D. Wohrle, Makromol. Chem. 175, 1751 (1974)

${ }^{48}$ S. C. Lind, D. C. Bordwell, and J. H. Perry, J. Am. Chem. Soc. 48, 1556 (1926).

${ }^{49}$ D. Jacquemin, E. A. Perpete, and J.-M. Andre, Int. J. Quantum Chem. 105, 553 (2005).

${ }^{50}$ M.-H. Whangbo, R. Hoffmann, and R. B. Woodward, Proc. R. Soc. London, Ser. A 366, 23 (1979).

${ }^{51}$ C. K. Chiang, C. R. Fincher, Jr., Y. W. Park, A. J. Heeger, H. Shirakawa, E. J. Louis, S. C. Gau, and A. G. Macdiarmid, Phys. Rev. Lett. 39, 1098 (1977).

${ }^{52}$ S. Bhattacharyya, Phys. Rev. B 70, 125412 (2004).

${ }^{53}$ C. Godet, Diamond Relat. Mater. 12, 159 (2003).

${ }^{54}$ I. S. Beloborodov, P. Zapol, D. M. Gruen, and L. A. Curtiss, Phys. Rev. B 74, 235434 (2006). 